compounds such as graphite bisulphate $\mathrm{C}_{24}{ }^{+} \mathrm{HSO}_{4}-2 \mathrm{H}_{2} \mathrm{SO}_{4}$, formed by electrochemical (or the equivalent chemical) oxidation in the presence of the appropriate acid molecules.

\section{Synthetic metals}

One basic reason for the current widespread interest in lamellar compounds stems from the fact that in these the partner molecules form charge transfer bonds with the carbon hexagon networks, which act as giant aromatic molecules in this respect. Such charge transfer bonding was first postulated some 25 years ago (McDonnell et al. J. chem. Soc. 191; 1951). Many examples have now been studied. The striking growth of research on these compounds seems to be due to their interest and promise as synthetic metallic conductors. Intercalate molecules in carbon lamellar compounds also show distinctive chemical behaviour as catalysts, and in other ways. Means of making various precise physical and optical measurements have become more refined in recent years, so that much more precise information is beginning to be available to help interpret the nature and properties of the charge transfer bands formed. Nearly one-third of some 60 papers discussed during the meeting dealt with this aspect. Practically all the advances described were very recent. Surprising new facts and novel theoretical arguments kept members of the conference in a ferment throughout.

\section{Inorganic chemistry}

Experimental methods were described for introducing intercalate species not hitherto known to form lamellar compounds, for example by the use of oxidising agents such as $\mathrm{C}_{6} \mathrm{~F}_{6} \mathrm{AsF}_{6}$ (N. Bartlett, Lawrence Berkeley Laboratory, University of California, Berkeley) or catalysts such as $\mathrm{Co}\left(\mathrm{AlCl}_{4}\right)_{2}(\mathrm{E}$. Stumpp, Technical University at Klausthal-Zellerfeld). Production of $\mathrm{C}_{5} \mathrm{Li}$ by interdiffusion of the powdered solid elements (Herold) is also a recent innovation in the use of $\mathrm{Li}$ as an electron donor intercalate. Formation of ternary lamellar compounds by intercalation of a second partner into binary lamellar compounds often occurs rather unexpectedly. Different instances described by several authors included the intercalation of molecular $\mathrm{H}_{2}$, of tetrahydrofurane, of benzene, or of other molecules into lamellar compounds with alkali metals. Apart from any effects on the local charge transfer bonds, such ternary intercalation usually pushes apart even funther the carbon hexagon layers from their initial separation of $3.35 \AA$ in graphite. This 'layer dissociation' may have important consequences for electrical and other properties of carbon lamellar compounds.

\section{Solid-state physics}

Clarification of physical properties of various lamellar compounds of graphite was discussed. There have been elegant advances in band theory and important information has been obtained by precision measurements of various optical properties. For example, it now seems clear from infrared and Raman spectra that the fractional charge transfer when molecular $\mathrm{Br}_{2}$ is intercalated is only a few tenths of one percent (G. Dresselhaus and M. S. Dresselhaus, Massachusetts Institute of Technology). Graphite/bromine was one of the first lamellar compounds for which a remarkable increase of metallic properties occurs on intercalation (McDonnell et al. op. cit.) and the rise in electrical conductivity begins steeply as the first few molecules of bromine enter the graphite. This recent optical work suggests (A. R. Ubbelohde, Imperial College, London) that metallic conduction in the halogen lamellar compounds resulting from charge transfer involves only relatively few additional charge carriers, but if so these must have exceptionally large mobilities.

Quite generally, under suitable conditions, synthetic metals formed by charge transfer in lamellar compounds of graphite seem to exhibit exceptionally low scattering of charge carriers travelling in the directions of the layer planes. This points to a two-dimensional mode of conduction which differs at least quantitatively and perhaps also qualitatively from that in the familiar three-dimensional metals (Ubbelohde). Such findings stress the importance of

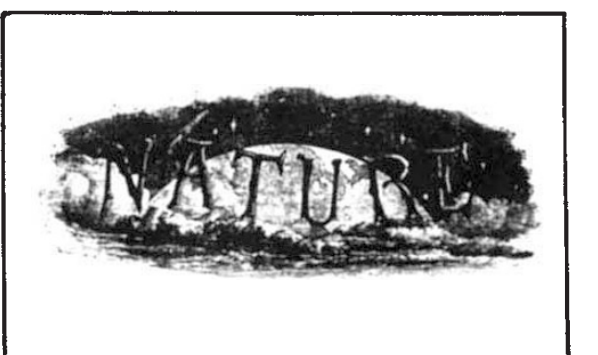

\section{A hundred years ago}

THE application of new materials for paper stock which has occupied so much attention of late seems to have attracted some notice in the Philadelphia Exhibition last year. From Jamaica bamboo was perhaps the most important paper-making plant exhibited. of the young bamboo stems, which are the best for the purpose, a very large supply, it is said, could be annually, by systematic cuttings or croppings, furnished from plants flourishing in the humid parts of the island.

from Nature 16, 5 July, 198; 1877. making many more measurements on the anisotropy of various physical properties parallel and perpendicular to the layer planes. Remarkably, very few $c$-axis measurements on lamellar compounds were presented to this conference, but there seems every hope that this gap will before long be filled.

\section{Ramsey fringes in optical spectroscopy}

\section{from Robin M. Hochstrasser}

EXPERIMENTS have been reported recently in which the spectral resolution in optical spectroscopy of gases can be limited only by the time of flight difference between two light pulses. Limitations due to Doppler broadening, the homogeneous width of the resonant state, or the transform spectral width of the laser pulse, have been eliminated.

The Doppler broadening of a spectral line at $\omega_{b a}$ can be eliminated if the transition $a^{-}>b$ is brought about by the simultaneous absorption of two photons, one from each of two counter-propagating beams having frequency $\omega_{b a} / 2$. This type of non-linear spectroscopy requires high light power so that present day laser beams must be sharply focused in order for the signals to become readily detectable. With high peak power pulsed lasers such as a nitrogen-pumped dye laser some focusing of the beam is still necessary to observe these non-linear effects.

A severe limitation of pulsed dye lasers in high resolution spectroscopy is that typical pulses have a duration of $8 \mathrm{~ns}$ corresponding to a minimum frequency uncertainty of $\sim 125 \mathrm{MHz}$ for transitions occurring during the transit of such a pulse. For molecules travelling at $10^{3} \mathrm{~m} \mathrm{~s}^{-1}$ across a laser beam focused to a diameter of $10^{-4} \mathrm{~m}$ the accuracy of the experiment is not limited by the transverse transit time which contributes only $\sim 10 \mathrm{MHz}$ to the spectral width. Transit time limitations on spectral accuracy have been known for a long time in beam resonance spectroscopy and in 1949 N. F. Ramsey (Molecular Beams, Ch V, Oxford University Press, 1956) suggested his method of separated oscillating fields in order to overcome this difficulty. In Ramsey's method the molecular beam passes through an RF field region of length $l$ in time $\tau$, and then travels a distance $L$ before reaching a second identical RF field region. The number of transitions at a fixed RF frequency undergone in such a spectrometer is an oscillatory function of the time $L / v$, where $v$ is the most probable velocity of molecules

Robin M. Hochstrasser is Professor of Chemistry at the University of Pennsylvania. 